\title{
Surgical treatment of gastric and small bowel gastrointestinal stromal tumours
}

\author{
Lukáš Sákra ${ }^{1,2}$, Jiři Šiller ${ }^{1,2}$, Petr Vyhnálek³, Marie Hácová4 \\ 1Faculty of Health Studies, University of Pardubice, Czech Republic \\ 2Surgical Department, General Hospital, Pardubice, Czech Republic \\ 3Internal Medicine Department, General Hospital, Pardubice, Czech Republic \\ 4Pathological Department, General Hospital, Pardubice, Czech Republic
}

Videosurgery and other miniinvasive techniques 2011; 6 (3): 138-143

DOI: 10.5114/wiitm.2011.24691

\begin{abstract}
Aim: This study aimed to evaluate a set of gastrointestinal stromal tumours (GIST) of the stomach and the small bowel managed with a laparoscopic technique.

Material and methods: The study covers a period from January 1, 2007 until June 1, 2010 during which 13 patients underwent the laparoscopic removal of stomach tumours and 2 patients underwent the removal of a small bowel GIST in the General Hospital in Pardubice. In all cases tumours were removed in a laparoscopic way, including the healthy border of the stomach tissue.

Results: No death was observed in our study. Two patients suffered from wound infection (secondary healing), one of them requiring repeat surgery owing to the excessive narrowing of the distal part of the stomach. Dehiscence of laparoscopic sutures or other intra-abdominal complications were not observed. During monitoring all patients were free of signs of local recurrence, but tumour progression into the liver was observed in 1 patient. Gastrointestinal stromal tumours are very rare tumours but their incidence is increasing. At this time the consensus about the necessity of preoperative unambiguous differentiation between malignant or less malignant variants is not available. Strict differentiation is very difficult and the decision whether to choose a more radical surgical approach for more malignant variants is not clear-cut.

Conclusions: In cases of gastric and small bowel GISTs the local removal of a tumour with the healthy border of the stomach tissue may be chosen as an adequate approach. Our results support this local surgical approach.
\end{abstract}

Key words: gastrointestinal stromal tumours, GIST, stomach laparoscopy, c-kit protein.

\section{Introduction}

This study aimed to evaluate a set of gastrointestinal stromal tumours (GIST) of the stomach and the small bowel treated by laparoscopic surgery and to consider an appropriate surgical technique in view of the nature of this type of tumour.

Benign tumours of the stomach and small bowel are quite rare. They include adenomas, which are found to be clearly pre-cancerous, lipomas, leiomyomata, neurogenic tumours (schwannomas, gangliotic paragangliomas, paragangliomas) and GISTs. The term gastrointestinal stromal tumour was introduced as a neutral name for a group of gastric mesenchymal tumours that can be classified as tumours of neurogenic origin or of smooth muscle [1]. The immunohistochemical proof of c-kit (CD 117) gene 
expression [2, 3] provides a generally recognized criterion for the inclusion of a respective mesenchymal tumour among GIST. The incidence of GIST has been on the increase over the past 10 years. In most cases GIST is accompanied by KIT and PDGFRA gene mutations that are responsible for the production of receptor tyrosine kinases. Therefore, these tumours produce growth factor receptors with tyrosine kinase activity. Very high tyrosine kinase activity in the malignant variants of this tumour leads to cell proliferation and resistance to apoptosis. It is the varying tyrosine kinase activity that creates a broad clinical spectrum of these tumours, ranging from benign to malignant forms. The immunohistochemical proof of tyrosine kinase in clinical and pathological practice differentiates GISTs from other mesenchymal tumours. Gastric GISTs arise from interstitial pacemaker Cajal cells or from poorly differentiated precursor cells (which develop into cells of Cajal). These cells are localized between the myenteric nerve plexus and the smooth muscle layer of the GIST wall $[4,5]$. These tumours are further characterized by resistance to chemotherapy, except imatinib mesylate.

Tumours of low malignant potential have a very good prognosis after surgical therapy alone (5-year survival rate $>90 \%$ ). On the other hand, tumours of high malignant potential have a bad prognosis despite the combination of surgical therapy and imatinib mesylate (median survival $<12$ months).

Considering the above-described behaviour and the limited choice of further therapy it is recommended to treat GIST by complete resection of the tumour without having to perform lymphadenectomy and wide resection edges [6, 7]. Laparoscopy appears to offer a highly appropriate method of removing GISTs [8-10]. Initial diagnosis is made by gastroscopy. In the case of gastric tumours biopsy samples are taken as well. It should be emphasized that save for rare exceptions biopsies taken during gastroscopy are negative. Therefore, the diagnostic algorithm must include endosonography and taking a biopsy sample from deeper layers. The overall picture and pre-operative staging is completed with a CT scan, whereas magnetic resonance imagining and stomach $\mathrm{X}$-ray with contrast dye are optional. The principal tools used to diagnose small bowel GISTs are CT and MRI. Enteroscopy is of low diagnostic value, which is due to its demanding nature and to the rather frequent extraluminal growth of the tumour.
Gastrointestinal stromal tumours represents 2-5\% of all gastrointestinal tract tumours. It metastasizes to the liver, soft tissues in the abdominal cavity (peritoneum and omentum) and retroperitoneum. Metastases in bones and lungs are very rare. On average, $20-25 \%$ of gastric and $40-50 \%$ of intestinal GISTs are malignant [11]. Smaller bowel GISTs have a more aggressive and malignant potential than stomach GIST. Moreover, these tumours are more aggressive in younger patients $[12,13]$.

The common symptoms of GIST are pain, bleeding into the gastrointestinal tract, anaemia and dyspepsia. According to professional literature the average survival rate ranges from 40.8 to 130.8 months, whereas the 5 -year survival rate for these patients is $53.9 \%$ to $74.7 \%$ [14-16]. The incidence of GIST reported in the Czech Republic between the years 2001 and 2005 was 0.52 patients per 100000 inhabitants. The average survival rate in the Czech Republic is 93.2 months and the 5-year survival rate is $78.3 \%$ [17].

\section{Material and methods}

The aim of this study was to evaluate a set of gastrointestinal stromal tumours (GIST) of the stomach and the small bowel treated by laparoscopic surgery and to consider an appropriate surgical technique in view of the nature of this type of tumour.

The study covers the period from January 1, 2007 until June 1, 2010 during which the General Hospital in Pardubice reported the laparoscopic excision of stomach tumours in 12 patients of whom 7 were treated for GIST. Two patients were operated on for small bowel GIST (see Table I).

\section{Stomach gastrointestinal stromal tumours}

The first 5 patients underwent pre-operative endoscopic tattooing. As peroperative gastroscopy had to be performed in all these patients to determine the precise localization of tumour edges, a decision was made not to carry out pre-operative tattooing in the remaining patients. Peroperative gastroscopy was applied in the event that the tumour was not visible after introducing a laparoscopic instrument. The inserted gastroscope could then be used to check the sufficiency of sutures. Except for two procedures when 5 ports were required, 4 ports were used during laparoscopies. A $10 \mathrm{~mm}$ camera port was inserted above the navel. $10 \mathrm{~mm}$ and $5 \mathrm{~mm}$ 
working ports were inserted on either side of the navel. One auxiliary $5 \mathrm{~mm}$ port was inserted in the left (and possibly also in the right) epigastrium. Afterwards, the tumour was laparoscopically removed with a harmonic scalpel and the laparoscopic suturing of the stomach wall defect was completed. The suturing was carried out in two lines; a continuous suture was used for the first line and an interrupted Vicryl suture for the other one. The sufficiency of the complete suturing was checked by means of a gastroscope. The localization of the tumour on the edge of the greater curvature enabled the wedge excision of a tumour by using two cartridges of the linear stapler only in one case. Every preparation was removed from the abdominal cavity in a polyethylene bag through a slightly extended excision above the navel.

The nasogastric probe was removed on the $2^{\text {nd }}$ to the $3^{\text {rd }}$ day after surgery. At the same time bowel passage was restored. Patients were discharged on the $3^{\text {rd }}$ to the $5^{\text {th }}$ day after surgery. Gastroscopic checkups including biopsy sampling and ultrasound imaging were performed 2 months and 6 months after surgery, whereas further check-ups are planned in 6 month intervals. Endosonographic check-ups were not carried out.

\section{Small bowel gastrointestinal stromal tumours}

Four ports were used: a camera port above the navel and 3 working ones (one $12 \mathrm{~mm}$ port and two $5 \mathrm{~mm}$ ports) in the left epigastrium, in the hypogastrium and above the pubic symphysis. In one case the

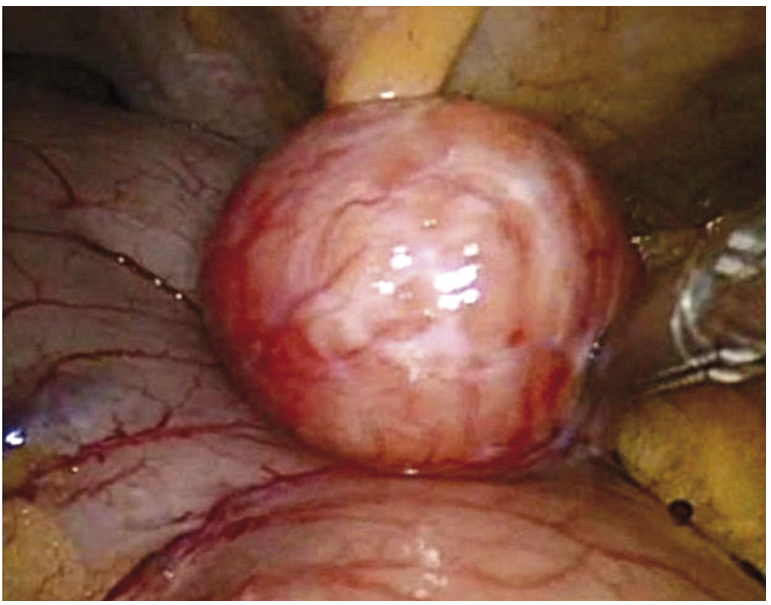

Figure 1. Peroperative picture of GIST of the stomach
GIST alone was removed with a linear stapler and in one case tumour size required converting the laparoscopic procedure into open surgery. The latter procedure involved small bowel resection.

\section{Results}

None of the patients included in the study group died in direct connection with the surgical procedure performed. Considering the malignant type of GIST 4 patients received imatinib mesylate therapy (3 patients were treated for a stomach tumour and 1 patient for a small bowel tumour). According to histological grading chemotherapy was not required in a patient suffering from leiomyoblastoma. The secondary healing of surgical wounds above the navel through which a preparation was removed was observed in 2 patients. In one case repeat surgery had to be performed because of the undue narrowing of the stomach antrum caused by the sutures applied after the removal of a tumour located too close to the pylorus. An open procedure was applied during repeat surgery (stomach resection, type BII, in Roux modification). Dehiscence or poor healing of laparoscopic suturing was not observed at all. The group of patients was free of any other complications. According to histological examinations none of the tumours reached as far as the edge of the excision line.

No local recurrence was observed during the monitoring stage. Overall progression of the tumour with multiple metastases in the liver occurred in one case where GIST had been assessed to have only

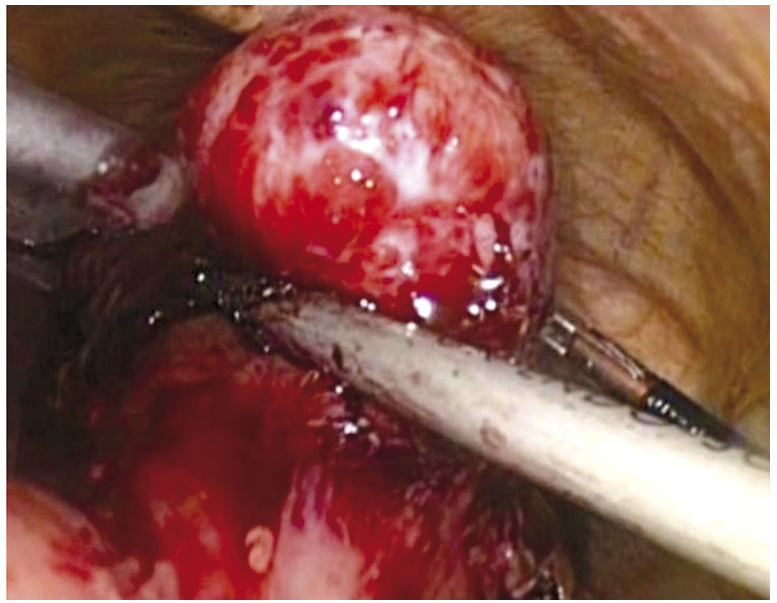

Figure 2. Peroperative picture of GIST of the stomach - resection 

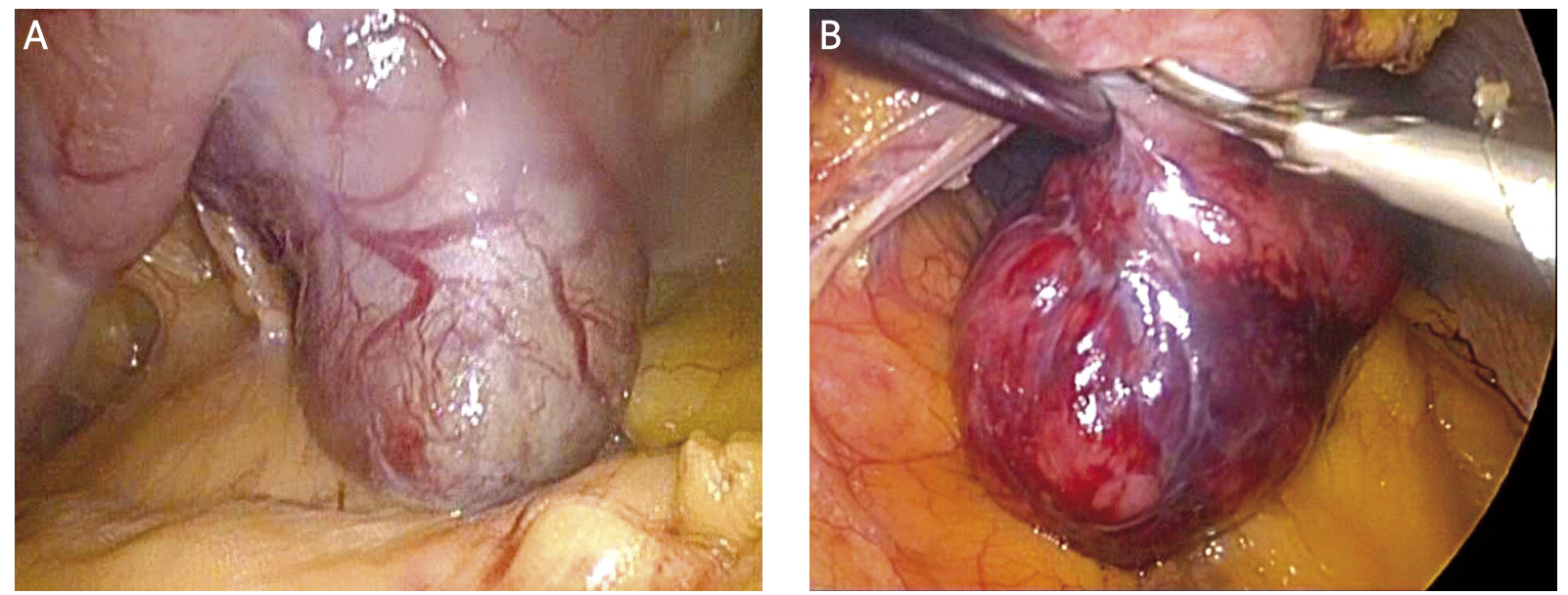

Figure 3 A-B. Peroperative picture of GIST of the distal stomach wall

a very low malignant potential and therefore the patient had not been treated with imatinib mesylate. All other patients were free of tumour progression, which also applied to those originally suffering from malignant GIST forms.

\section{Discussion}

Theoretically, pre-operative gastroscopic tattooing along with the other imaging methods should suffice to localize a stomach tumour and its edges for the purpose of a laparoscopic approach. In practice, tattooing was confirmed not to be of great significance. In the case of large tumours this procedure is not necessary and in minor tumours and in carcinomas in situ it is inaccurate. Unlike in the case of tattooing of the intestine, the dye becomes blurred due to the abundant lymphatic drainage of the stomach wall and cannot be used to determine tumour edges. When localizing indistinct margins peroperative gastroscopic localization proved effective given the current laparoscopic knowledge available. After completing the procedure the gastroscope should also be used to check the sufficiency of sutures and stomach patency.

Preoperative endosonography is an essential and determining examination whose performance is absolutely necessary. This method evaluates tumour boundaries in the stomach, yet the biopsy taken during endosonography also has many benefits. Due to the intact mucosa of the stomach biopsies taken during gastroscopy are almost always negative. Often an experienced endosonographer can precisely assess
Table I. Histological description of laparoscopically operated tumours

\begin{tabular}{|c|c|}
\hline Histological type & Number \\
\hline Gastric GIST & $\begin{array}{l}7 \text { (3 occurrences } \\
\text { of high malignant potential) }\end{array}$ \\
\hline Small bowel GIST & $\begin{array}{l}2 \text { (1 occurrence } \\
\text { of high malignant potential) }\end{array}$ \\
\hline Gastric adenoma & 1 \\
\hline Gastric leiomyoma & 2 \\
\hline Gastric schwannoma & 1 \\
\hline Gastric leiomyoblastoma & 1 \\
\hline Atopic pancreas in stomach & 1 \\
\hline
\end{tabular}

the nature and type of tumour on the basis of the sonographic scan alone.

Laparoscopic excisions should be performed in facilities that have expertise with laparoscopic surgery of the upper passage of the gastric tube. Tumours located on the distal stomach wall are rather difficult to remove. A transgastric approach complemented with gastrotomy of the proximal stomach wall is considered to be too complicated by the authors of this paper. Upon mobilizing the proximal stomach curvature, opening the omental bursa and elevating the proximal stomach wall, removal of a tumour from the distal stomach wall can be completed without major difficulties [8, 9].

Surgical procedures applied in the case of small bowel GIST are straightforward. The spread of the tumour is almost always extramural and therefore it 
can be easily removed with a linear stapler with the adjacent part of the small bowl wall. Most tumours require resection of a part of the small bowel. In these cases minor laparotomy is applied to remove the tumour and small bowel resection can thus be performed without major difficulties after luxating the tumour along with the small bowel in front of the abdominal wall.

According to professional literature the excision of a tumour with wide borders (using an open or laparoscopic approach) should be sufficient even when laparoscopy is applied. This clearly holds true for histologically confirmed adenomas, leiomyomata and other benign mesenchymal tumours [7, 10]. However, GIST of the stomach could present a more complex issue. Given the inadequate number of patients included in previously studied groups and primarily the very difficult comparison of individual patients owing to the highly diverse histological composition of tumours, determination of the optimum surgical procedure remains demanding and is hard to define during the pre-operative stage. It is the more detailed determination of pre-operative grading that poses the biggest problem when planning an appropriate surgical procedure. The samples of mucosa taken during gastroscopy show malignant elements only in a very small number of patients. Samples taken during endosonography are processed cytological ones and the determination of tyrosine kinase activity is rather problematic $[2,3,11,12]$. Therefore, the clear identification of which tumour has a high malignant potential is uncertain during the pre-operative stage. The same applies to the use of frozen peroperative histological methods. However, if we admit that some surgically treated tumours may be of high malignant potential, would a more radical approach not be more appropriate? If we managed to prove a high malignant potential during the preoperative stage in a clear and indisputable way, it would be advisable to consider stomach resection as an adequate option. However, in view of the above-presented facts and the results of available, yet not too large studies, local excision appears to be a suitable and adequate procedure. The results obtained by the authors of this paper support this statement.

\section{Conclusions}

Considering the increasing incidence of GISTS, this type of tumour has be paid undivided attention.
The laparoscopic approach appears to be highly appropriate in most patients. In the case of GIST of the stomach a completely laparoscopic approach can be applied to remove a stomach wall tumour with tumour-free margins. From an oncological standpoint, this local surgical approach that does not require lymphadenectomy is found to be sufficiently radical even in cases of malignant GIST variants. Our results clearly support this statement.

Gastrointestinal stromal tumours of the small bowel can be larger in size. As a result the main purpose of laparoscopy is to locate the tumour and to determine the site where to perform minor laparotomy to luxate the small bowel with the tumour in front of the abdominal wall.

\section{Acknowledgments}

The authors do not have any financial interest in the methods, results, commercial devices, equipment, instruments, or drugs described in this study.

\section{References}

1. Mazur MT, Clark HB. Gastric stroma tumors: reappraisal of histogenesi. Am J Surg Pathos 1983; 7: 507-19.

2. Sarlomo-Rikala M, Kovatich A, Barusevicius A, et al. CD 117: a sensitive marker for gastrointestinal stroma tumors that is more specific than CD 34. Mod Pathol 1998; 11: 728-34.

3. Miettinen M, Sarlomo-Rikala M, Lasota J. Recent advances in understanding of thein biology. Hum Patol 1999; 10: 1213-8.

4. Mucciarini C, Rossi G, Bertolini F, et al. Incidence and clinicopathologic features of gastrointestinal stroma tumors. A population-based study. BMC Cancer 2007; 230: 1186.

5. Nilsson B, Bumming P, Meis-Kindblom JM, et al. Gastrointestinal stromal tumors: the incidence, prevalence, clinical course, and prognostication in the preimatinib mesylate era. A population based study in western. Cancer 2005; 103: 821-9.

6. Wu T, Lee $\mathrm{L}$, Yeh $\mathrm{CH}$, et al. Surgical treatment and prognostic analysis for gastrointestinal stroma tumor sof the small intestine: efore era of imatinib mesylate. BMC Gastroenterol 2006; 29: 323-41.

7. Wente M, Buchler M, Weitz J. Gastrointestinal stromal tumors (GIST). Surgical therapy. Chirurgia 2008; 7: 638-43.

8. Warsi A, Peyser P. Laparoscopic resection of gastric GIST and benign gastric tumors: evolution of a new technique. Surg Endosc 2009; 9: 561-9.

9. Basso N, Rosato P, De Leo A, et al. Laparoscopic treatment of gastric stromal tumors. Surg Endosc 2000; 6: 524-6.

10. Everett M, Gutman H. Gastrointestinal stromal tumors. Surg Oncol 2008; 15: 588-93.

11. Miettinen M, Lasota J. Gastrointestinal stroma tumors: review on morphology, molecular pathology, prognosis, and differential diagnosis. Arch Pathol Lab Med 2006; 130: 1466-78. 
12. Rabin I, Chilkman B, Lavy R, et al. Gastrointestinal stromal tumors: a 19 year experience. IMAJ 2009; 12: 98-102.

13. Rutkowski P, Nowecki ZI, Michej W, et al. Risk criteria and prognostic factors for predicting recurrences after resection of primary gastrointestinal stromal tumors. Ann Surg Oncol 2007; 14: 2018-27.

14. Rubió J, Narcis-Gragera R, Ortiz MR, et al. Population based incidence and survival of gastrointestinal stroma tumors (GIST) in Gerona, Spain. Eur J Cancer 2007; 43: 144-8.

15. Brabec P, Sufliarsky J, Linke Z, et al. A whole population study of gastrointestinal stroma tumors in the Czech Republic and Slovakia. Neoplasma 2009; 5: 459-63.

16. Buchler P, Morel P. Gastrointestinal stromal tumors (GIST). Rev Med Suisse 2008; 4: 1567-70

17. Miettinen M, Lasota J. Gastrointestinal stromal tumors- definition, clinical, histological, imunohistochemical and molecular genetic features and differential diagnosis. Virchov Arch 2001; 438: 1-12. 